EPJ Web of Conferences 81, 02023 (2014)

DOI: $10.1051 /$ epjconf/201481 02023

(C) Owned by the authors, published by EDP Sciences, 2014

\title{
Poles in the SAID NN analysis
}

\author{
Ron Workman ${ }^{1, a}$ \\ ${ }^{1}$ Institute for Nuclear Studies, The George Washington University, Washington, DC
}

\begin{abstract}
The SAID approach to partial-wave analysis of nucleon-nucleon scattering data is reviewed. This has been applied to recent COSY $n p$ scattering data. The appearance of poles (dibaryons) is discussed in light of these new fits.
\end{abstract}

\section{Introduction}

Recent and precise polarization data $\left(A_{y}\right)$ from the COoler SYnchrotron (COSY) at Jülich have provided motivation for renewed $N N$ partial-wave analyses at medium energies. The sets of $p p$ measurements at COSY-ANKE cover a wide energy range ( $T_{p}$ from 0.8 to $2.4 \mathrm{GeV}$ ), are more precise than existing data, and cover forward angles at which no previous measurements exist. Significant deviations from the SAID fit [1] (SP07) suggest changes will be necessary in some isovector amplitudes.

Measurements of $n p A_{y}$ from the WASA-at-COSY collaboration [2] are also problematic for the SAID fit of 2007. In this case, however, the problem appears to be significant over a fairly narrow range of energies. The angular set in question corresponds to a center-of-mass energy of $2.4 \mathrm{GeV}$, which matches the peak of the purported "ABC" (Abashian-Booth-Crowe) effect noted by the WASA collaboration in the $p n \rightarrow d \pi^{0} \pi^{0}$ reaction [3]. From the analysis of this measurement, a narrow resonance with $I\left(J^{P}\right)=0\left(3^{+}\right), \Gamma=70 \mathrm{MeV}$ was announced. This result has spawned a number of theoretical calculation claiming to explain such a state [4].

We note that a resonance with these quantum numbers was predicted in early SU(6) [5] and quarkmodel studies [6], with the expectation of a strong coupling to $\Delta \Delta$. Previous (dibaryon) resonance claims have been mainly associated with poles found in the vicinity of the $N \Delta$ cut. The difficulty in distinguishing between "true" and "pseudo" resonances [7], producing similar effects, led most to conclude the evidence for their existence was insufficient. The present case differs in having a structure with a much smaller width and nearby branch points associated with he $\Delta \Delta$, and possibly the $N N(1440)$, production thresholds [8].

Much of the experimental evidence from the WASA collaboration is covered in the plenary talk of Heinz Clement at this conference. Below, I will just outline the results of a fit to these new $n p$ data within the present SAID NN analysis approach.

\section{Fits to the WASA-at-COSY $n p$ data}

The starting point for these fits is a partial-wave solution published [1] in 2007 (SP07), with a form based on the product of "exchange" and "production" S-matrices. The exchange piece is parameter-

a e-mail: rworkman@gwu.edu 
ized via a K-matrix and contains a one-pion exchange piece plus a sum over expansion bases containing a left-hand cut. The production piece is parameterized in terms of a Chew-Mandelstam K-matrix and allows for the production of an inelastic channel. For isovector waves, the inelasticity is assumed to be dominated by the $N \Delta$ channel. For isoscalar waves, there is an effective second channel. The formalism for both spin-coupled and uncoupled waves is explained in detail in Ref. [9].

This formalism leads to poles in the isovector waves - in particular the ${ }^{1} D_{2}$ partial wave. Resonance-like structures associated with this wave have also been noted in the SAID fits to $\pi d$ elastic scattering [10] and the reaction $\pi d \rightarrow p p$ [11]. This is important, as the existence of a ${ }^{1} D_{2}$ dibaryon state is assumed in the calculation of Ref. [4]. However, prior to the present study, no evidence for a pole had been noted in any SAID isoscalar partial-wave amplitudes.

The WASA-at-COSY data were entered into the full database as angular distributions at kinetic energies of 1108, 1125, 1135, 11391171 , and $1197 \mathrm{MeV}$, covering a fairly narrow range of energies. These data were not well-predicted by the SP07 solution, giving a chi-squared/datum of approximately 1300/68. An initial fit, using the original SP07 functional form, yielded a "reasonable" chi-squared/datum (1.8) for all energies apart from $1135 \mathrm{MeV}$, which retained a very high value (a chi-squared/datum of $\sim 25$ ).

In order to "force" a fit to this angular distribution, the statistical errors were reduced (by a factor of 4), and parameters were added to the inelastic channel coupling. This resulted in a solution with a much improved fit to the $1135 \mathrm{MeV}$ data which simultaneously improved the fit to the new data sets at other energies. The revised fit had significant changes in the coupled ${ }^{3} D_{3}-{ }^{3} G_{3}$ waves, with other waves remaining relatively stable. A subsequent fit, using the new parameter placement, and using proper statistical uncertainties for the $1135 \mathrm{MeV}$ dataset, resulted in a fit with a qualitatively similar description of the data, and with a resonance-like behavior appearing in the ${ }^{3} D_{3}$ partial wave in particular.

Some prior evidence for the resonance-like behavior of the ${ }^{3} D_{3}$ partial-wave amplitude can be seen in the single-energy solutions (SES) associated with this wave. The last available SES exhibits a rise in the imaginary part and a drop through zero for the real part - a behavior not seen in the underlying, energy-dependent solution SP07. The revised fit follows this behavior quite closely. Recall that SES fits have been done in order to search for systematic deviations from the energy-dependent solution. Unfortunately, this is the last SES due to the fact that the $n p$ database becomes very sparse above approximately $1.3 \mathrm{GeV}$. The SP07 and revised fits to $n p$ scattering data have extended up to $2 \mathrm{GeV}$, but the isoscalar waves are largely unconstrained above $1.3 \mathrm{GeV}$. The isovector waves are determined from $p p$ scattering data up to $3 \mathrm{GeV}$.

\section{Pole structure in the fit}

The behavior seen in the ${ }^{3} D_{3}$ partial wave suggests an s-channel resonance is present. This is confirmed both by examining the complex plane structure, as was done for isovector waves, or through the use of a simple speed-plot analysis. The variation in determinations is reflected in the pole position $(2380 \pm 10)-i(40 \pm 5) \mathrm{MeV}$. Unlike the poles found in isovector waves, the pole and possibly-dominant branch points are not coincident in this case. It is therefore more difficult to assign this behavior to a pseudo-resonance.

The certainty of this resonance hypothesis would be greatly increased with further polarization measurements in the vicinity of, and above, the observed structure. There is presently little data to distinguish the very-different behaviors of the SP07 and revised fits above $1.2 \mathrm{GeV}$, as shown in figure 1. 


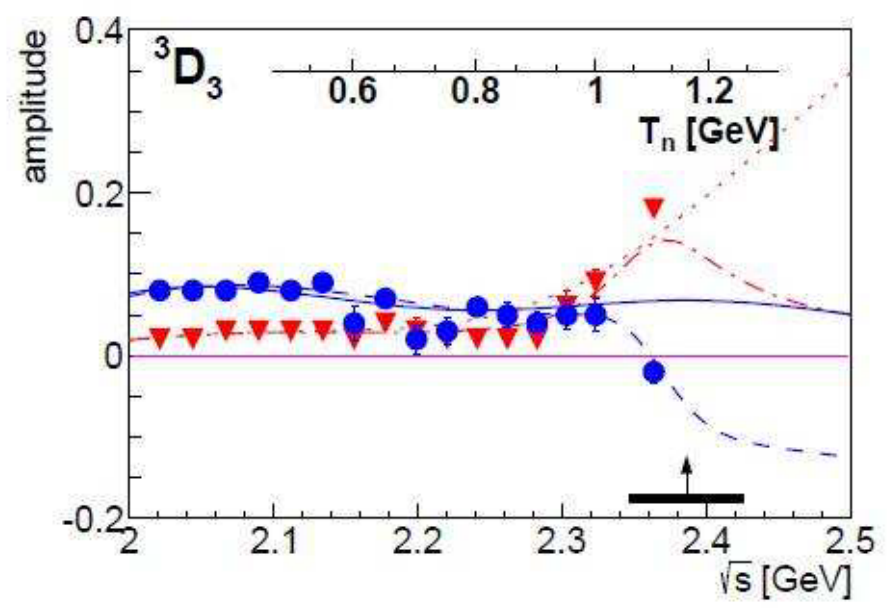

Figure 1. Comparison of fit SP07 and a revision including WASA data. SES are denoted by triangles (filled circles) for imaginary (real) parts. Real (imaginary) part of SP07 plotted as a solid (dotted) line. Real (imaginary) part of revised fit plotted as a dashed (dot-dashed) line.

\section{Acknowledgements}

The author acknowledges helpful discussions with M. Bashkanov, H. Clement, A. Gal, and Z.Y. Zhang regarding the WASA-at-COSY data and their interpretation. This work has been supported by a U.S. Department of Energy Grant No. DE-FG02-99ER41110.

\section{References}

[1] R.A. Arndt, W.J. Briscoe, I.I. Strakovsky, R.L. Workman, Phys. Rev. C76, 025209 (2007).

[2] P. Adlarson et al., Phys. Rev. Lett. 112, 202301 (2014).

[3] P. Adlarson et al., Phys. Rev. Lett. 106, 242302 (2011).

[4] A. Gal and H. Garcilazo, Phys. Rev. Lett. 111, 172301 (2013).

[5] F. Dyson and N.-H. Xuong, Phys. Rev. Lett. 13, 815 (1964).

[6] See, for example, M. Bashkanov, S. Brodsky, and H. Clement, Phys. Lett. B 727, 438 (2013); T. Goldman, K. Maltman, G.J. Stephenson, K.E. Schmidt, and F. Wang, Phys. Rev. C 39, 1889 (1989); X.Q. Yuan, Z.Y. Zhang, Y.W. Yu, and P.N. Shen, Phys. Rev. C 60, 045203 (1999).

[7] B.L.G. Bakker, I.M. Narodsky, and Yu. A. Simonov, Nuo. Cim. 19, 265 (1977).

[8] Difficulties in distinguishing between resonance poles and complex branch points appear in other reactions; see for example, A. Svarc et al., Phys. Rev. C 89, 065208 (2014).

[9] R.A. Arndt, J.S. Hyslop, L.D. Roper, Phys. Rev. D 35, 128 (1987); R.A. Arndt, C.H. Oh, I.I. Strakovsky, R.L. Workman, F. Dohrmann, Phys. Rev. C 56, 3005 (1997).

[10] R.A. Arndt, I.I. Strakovsky, and R.L. Workman, Phys. Rev. C 50, 1796 (1994).

[11] R.A. Arndt, I.I. Strakovksy, R.L. Workman, and D.V. Bugg, Phys. Rev. C 48, 1926 (1993). 